Original Research

\title{
Inpatient versus outpatient shoulder arthroplasty outcomes: A propensity score matched risk-adjusted analysis demonstrates the safety of outpatient shoulder arthroplasty
}

\author{
Maxwell T. Trudeau, BS *, Jonathon J. Peters, BS, Matthew R. LeVasseur, MD, \\ Benjamin C. Hawthorne, BS, Caitlin G. Dorsey, BS, Ian J. Wellington, MD, Kevin P. Shea, MD, \\ Augustus D. Mazzocca, MS, MD \\ Department of Orthopaedic Surgery, University of Connecticut School of Medicine, Farmington, CT, USA
}

\section{A R T I C L E I N F O}

\section{Keywords:}

Shoulder arthroplasty

NSQIP

Inpatient

Outpatient

Outcomes

Adverse outcomes

\begin{abstract}
A B S T R A C T
Objectives: Beginning January 1, 2021 total shoulder arthroplasty (TSA) was removed from the Medicare (U.S national healthcare for patients $\geq 65$ years of age) inpatient-only list. Furthermore, there is limited data comparing outpatient and inpatient TSA among recent contemporary large population databases. This study aimed to analyze shoulder arthroplasty outcomes between inpatient and outpatient procedures at the national level.

Methods: The American College of Surgeons National Surgical Quality Improvement Program (ACS-NSQIP) database was utilized (2015-2019). Cases with a current procedural terminology of 23472 indicative of primary TSA and reverse total shoulder arthroplasty were included $(\mathrm{N}=22,452)$. Outcomes were then analyzed in two approaches: unmatched analysis and propensity score matched risk-adjusted analysis.

Results: Overall, 9.7\% ( $\mathrm{N}=2,185)$ of cases were performed outpatient and $90.3 \%(\mathrm{~N}=20,357)$ of cases were performed inpatient. The rate of outpatient procedures has been steadily increasing (2015: 8.3\%, 2016: 14.7\%, 2017: 15.8\%, 2018: 26.5\%, 2019: 34.6\%; P < 0.001). Outpatients were more likely to be male (50.7\% vs. $43.7 \%)$ and younger (age $<65 ; 37.0 \%$ vs. $27.9 \%$ ) and less likely to be ASA class 3 or 4 (49.5\% vs. 58.3\%). Outpatients had fewer comorbidities including obesity (46.1\% vs. $51.9 \%)$, hypertension $(60.5 \%$ vs. $67.4 \%)$, diabetes (15.1 vs. $18.2 \%$ ), chronic obstructive pulmonary disease ( $4.8 \%$ vs. $7.0 \%)$, bleeding disorders (1.3\% vs. $2.5 \%)$, or chronic steroid use $(3.5 \%$ vs. $5.0 \%$; all $\mathrm{P}<0.001)$. In a non-risk matched analysis of outcomes, outpatient procedures displayed lower rates of any adverse event (3.5\% vs. 5.3\%; P $<0.001)$, minor adverse events $(1.5 \%$ vs. $3.0 \%$; $\mathrm{P}=0.001)$, and readmission $(2.2 \%$ vs. $2.8 \% ; \mathrm{P}=0.025)$. Following a propensity score matched analysis, two risk matched cohorts of outpatient $(\mathrm{N}=2,172)$ and inpatient $(\mathrm{N}=2,172)$ procedures were identified. Subsequent analysis of outcomes revealed no significant differences in outcome metrics between risk-matched outpatient and inpatient procedures.

Conclusions: From 2015 to 2019, there has been a four-fold increase in the proportion of outpatient shoulder arthroplasty cases in the ACS-NSQIP database. This study shows that outpatient shoulder arthroplasty may be safely performed in a select cohort of patients without increased risk of adverse events. After adjusting for comorbidities, there were no differences in clinical outcomes or rates of adverse outcomes between inpatient and outpatient shoulder arthroplasty.

Level of evidence: Retrospective Observational Study, Level IV.
\end{abstract}

\footnotetext{
* Corresponding author. Tel.: +(203)-606-8630.

E-mail address: trudeau@uchc.edu (M.T. Trudeau).
} 


\section{What are the new findings?}

- Shoulder arthroplasty can be safely performed in the outpatient setting with equivalent outcomes.

- In the past 5 years, the proportion of outpatient shoulder arthroplasty cases in the NSQIP database has increased four-fold (8.3\%-34.6\%).

- Currently, patients being selected for outpatient shoulder arthroplasty have significantly less comorbidities.

\section{Introduction}

Over the past decade, outpatient total shoulder arthroplasty has become increasingly popular, with four-fold increases over just the past five years [1]. More importantly, on January 1, 2021 total shoulder arthroplasty was removed from the Medicare (U.S national healthcare for patients $\geq 65$ years of age) inpatient-only list, which may be the start of increased same-day discharge for private insurance as well [2]. In 2008, Gallay et al. investigated the feasibility of outpatient shoulder arthroplasty in a pilot study and, demonstrated improved pain and patient satisfaction scores with decreased readmissions [3]. This paradigm shift in shoulder arthroplasty has been accelerated due to advances in multimodal anesthesia techniques, effective venous thromboembolism prophylaxis, and appropriate blood and wound care management [4]. The safety and efficacy of outpatient shoulder arthroplasty has been demonstrated in many single-institutional experiences [5-14], large national database investigations [15-18], and systematic reviews with meta-analyses [19-22]. Furthermore, many studies have reported the potential financial advantages of performing outpatient shoulder arthroplasty, predicting annual savings of approximately $\$ 100$ million for the healthcare system [4,23-25]. Other investigations have defined objective selection criteria for determining which patients are the best candidates for outpatient shoulder arthroplasty [26-28].

However, few studies have utilized the American College of Surgeons National Surgical Quality Improvement Program (ACS-NSQIP) database to analyze outpatient shoulder arthroplasty outcomes [15]. Steinhaus et al. used the NSQIP database in an attempt to identify patients not suitable for same day discharge, but did not provide any outcomes comparisons between outpatient and inpatient [17]. To date, the only existing NSQIP investigation comparing outpatient and inpatient complications included patients from 2005 to 2014 and was limited to 173 outpatient procedures [16]. Moreover, recent survey data demonstrates that less than $25 \%$ of surgeons perform outpatient shoulder arthroplasty [4]. There is a paucity of data that exists analyzing rates of contemporary outpatient shoulder arthroplasty. Therefore, this study aimed to (1) analyze shoulder arthroplasty outcomes between inpatient and outpatient procedures, and (2) define current rates in outpatient shoulder arthroplasty procedures at the national level.

\section{Methods}

Total shoulder arthroplasty and reverse shoulder arthroplasty procedures were queried using the Current Procedural Code (CPT) 23472 to identify cases within the American College of Surgeons National Surgical Quality Improvement Program (ACS-NSQIP). The ACS-NSQIP database utilized consisted of five years of data (2015-2019) from over 700 academic and community hospitals. Preoperative, intraoperative, and postoperative factors were included in the database. Patient characteristic data included age, gender, race, American Society of Anesthesiologist (ASA) classifications, smoking, obesity, hypertension, diabetes mellitus, congestive obstructive pulmonary disease (COPD), congestive heart failure (CHF), weight loss greater than $10 \%$ within 6 months prior to surgery, bleeding disorders, steroid use, and operative time. Clinical outcome data included superficial incisional surgical site infections, deep incisional surgical site infections, organ/space surgical site infections, wound disruption, pneumonia, unplanned intubation, pulmonary embolism, ventilator use, progressive renal insufficiency, acute renal failure, urinary tract infection, stroke, cardiac arrest, myocardial infarction, transfusions, deep vein thrombosis, sepsis, septic shock, and Clostridium difficle infections (all within 30 days post-operation). Composite outcome metrics were then categorized into severe, minor and any adverse event based on previous research [16]. Severe adverse events included 30-day mortality, cardiac arrest, deep vein thrombosis, myocardial infarction, post-operative intubation, ventilator use, acute renal failure, pulmonary embolism, surgical site infections (superficial, deep, and organ/space), reoperation, stroke, and sepsis. Minor adverse events included progressive renal insufficiency, wound disruption, pneumonia, urinary tract infection, and blood transfusion. Any adverse event consisted of both severe and minor adverse events. This study analyzed outcomes using two approaches: (1) unmatched analysis and (2) propensity score matched risk-adjusted analysis.

\section{Statistical analysis}

Descriptive statistics encompassing categorical variables were presented as frequencies and percentages. The association between categorical variables was tested using Pearson's $\chi 2$ test or Fisher exact test, as appropriate. Continuous variables were expressed as mean with standard deviation when parametric and medians and interquartile ranges (IQR) when non-parametric, and comparisons among groups were conducted through ANOVA test or Kruskal-Wallis test, as appropriate. All tests were 2-sided and type I error of 0.05 was used for analysis. Analyses were performed with SPSS 27.0 (IBM Corporation, Armonk, NY).

Propensity score matching (PSM) is a technique accounting for treatment selection bias when estimating treatment effects using nonrandomized retrospective data. This study utilized a multivariable logistic regression model to calculate propensity scores accounting for patient characteristics that were significantly different between inpatient and outpatient shoulder arthroplasty procedures (sex, age, ASA, obesity, hypertension, COPD, bleeding disorders, and chronic steroid use) [29]. In this framework, a propensity score was calculated for the patient's probability of receiving a total shoulder arthroplasty inpatient or outpatient. After propensity score calculation, outpatient cases were paired with controls (inpatient) 1:1 using an exact matching algorithm without replacement. Exact matching (caliper $=0.0$ ) results in an unbiased balance of covariates, although reducing the number of matched subjects. After 1:1 matching, outcomes were re-analyzed.

\section{Results}

\section{Baseline patient characteristics}

Overall, 9.7\% $(\mathrm{N}=2,185)$ of cases were performed outpatient and $90.3 \%(N=20,357)$ of cases were performed inpatient. Patients who had outpatient procedures were more likely to be male $(50.7 \%$ vs. $43.7 \%$; $\mathrm{P}<0.001$ ) and younger (age $<65 ; 37.0 \%$ vs. $27.9 \%$ ). Outpatients were also less likely to be ASA class 3 or $4(49.5 \%$ vs. $58.3 \%$; $P<0.001)$ and had fewer comorbidities including obesity ( $46.1 \%$ vs. $51.9 \%$; $<0.001$ ), hypertension $(60.5 \%$ vs. $67.4 \%$; $\mathrm{P}<0.001)$, diabetes $(15.1$ vs. $18.2 \%$; $\mathrm{P}<0.001)$, chronic obstructive pulmonary disease $(4.8 \%$ vs. $7.0 \%$; $\mathrm{P}<0.001)$, bleeding disorders $(1.3 \%$ vs. $2.5 \%$; $\mathrm{P}<0.001)$, or chronic steroid use $(3.5 \%$ vs. $5.0 \%$; $<0.001)$. Further details between the two cohorts can be observed in Table 1 .

\section{Outcomes}

In a non-risk matched analysis of outcomes, outpatient procedures displayed lower rates of any adverse event ( $3.5 \%$ vs. $5.3 \%$; $<0.001)$, minor adverse events (1.5\% vs. $3.0 \%$; $=0.001)$, and readmission $(2.2 \%$ vs. $2.8 \%$; $\mathrm{P}=0.025$; Table 2 ). 
Table 1

Comparison of patient characteristics between inpatient and outpatient shoulder arthroplasty procedures $(\mathrm{N}=22,452)$.

\begin{tabular}{|c|c|c|c|c|c|}
\hline \multirow[t]{2}{*}{ Variables } & \multicolumn{2}{|c|}{$\begin{array}{l}\text { Inpatient } \\
\mathrm{N}=20,357\end{array}$} & \multicolumn{2}{|c|}{$\begin{array}{l}\text { Outpatient } \\
\mathrm{N}=2185\end{array}$} & \multirow[t]{2}{*}{$\mathrm{P}$} \\
\hline & $\mathrm{N}$ & $\%$ & $\mathrm{~N}$ & $\%$ & \\
\hline \multicolumn{6}{|l|}{ Gender } \\
\hline Female & 11453 & $56.3 \%$ & 1078 & $49.3 \%$ & \multirow[t]{2}{*}{$<0.001$} \\
\hline Male & 8904 & $43.7 \%$ & 1107 & $50.7 \%$ & \\
\hline \multicolumn{6}{|l|}{ Race } \\
\hline $\begin{array}{c}\text { Black/African } \\
\text { American }\end{array}$ & 965 & $4.7 \%$ & 112 & $5.1 \%$ & \multirow[t]{3}{*}{0.643} \\
\hline White & 17057 & $83.8 \%$ & 1831 & $83.8 \%$ & \\
\hline Other & 2335 & $11.5 \%$ & 242 & $11.1 \%$ & \\
\hline \multicolumn{5}{|l|}{ ASA } & \multirow[t]{3}{*}{$<0.001$} \\
\hline $1 \& 2$ & 8479 & $41.7 \%$ & 1104 & $50.5 \%$ & \\
\hline $3 \& 4$ & 11878 & $58.3 \%$ & 1081 & $49.5 \%$ & \\
\hline Age $\geq 65$ years & 14593 & $72.1 \%$ & 1372 & $63.0 \%$ & $<0.001$ \\
\hline Obesity & 10575 & $51.9 \%$ & 1008 & $46.1 \%$ & $<0.001$ \\
\hline Hypertension & 13725 & $67.4 \%$ & 1321 & $60.5 \%$ & $<0.001$ \\
\hline Diabetes mellitus & 3699 & $18.2 \%$ & 330 & $15.1 \%$ & $<0.001$ \\
\hline COPD & 1416 & $7.0 \%$ & 105 & $4.8 \%$ & $<0.001$ \\
\hline Congestive heart failure & 147 & $0.7 \%$ & 8 & $0.4 \%$ & 0.056 \\
\hline Weight loss $(>10 \%)$ & 37 & $0.2 \%$ & 5 & $0.2 \%$ & 0.628 \\
\hline Bleeding disorders & 512 & $2.5 \%$ & 29 & $1.3 \%$ & 0.001 \\
\hline Steroid use & 1022 & $5.0 \%$ & 76 & $3.5 \%$ & 0.001 \\
\hline
\end{tabular}

ASA, American Society of Anesthesiologists.

Bold indicates significance.

Following a propensity score matched analysis, two risk-matched cohorts of inpatient $(\mathrm{N}=2,172)$ and outpatient $(\mathrm{N}=2,172)$ procedures were identified (Table 3). All previous differences in patient characteristics (gender, ASA class, age, obesity, hypertension, diabetes, COPD, bleeding disorders, and steroid use) were successfully balanced (all $\mathrm{P}=1.000$ ). In this risk-matched comparison there were no significant differences in any adverse event (3.5\% vs. $4.1 \%$; $\mathrm{P}=0.339)$, minor adverse event ( $1.5 \%$ vs. $2.0 \% ; \mathrm{P}=0.200$ ), severe adverse event ( $2.3 \%$ vs. $2.5 \% ; \mathrm{P}=0.551)$, readmission $(2.1 \%$ vs. $2.6 \% ; \mathrm{P}=0.229)$, or reoperation $(1.0 \%$ vs. $1.7 \% ; \mathrm{P}=0.066)$. While not statistically significant, the outpatient cohort had lower rates of adverse outcomes compared to the inpatient group (any adverse event $=3.5 \%$ vs. $4.1 \%$; Table 4 ).

\section{Current trends in shoulder arthroplasty}

Overall, when focusing on inpatient procedures, length of stay has decreased over recent years; 2015: $1.9 \pm 3.6$ days, 2016: $1.8 \pm 3.6$ days, 2017: $1.7 \pm 3.1$ days, 2018: $1.4 \pm 4.2$ days, 2019: $1.3 \pm 3.2$ days ( $\mathrm{P}<0.001$ ). Median and IQR for length of stay by year demonstrated similar findings; 2015: 2.0 (IQR: 1.0-2.0), 2016: 1.0 (IQR: 1.0-2.0), 2017: 1.0 (IQR: 1.0-2.0), 2018: 1.0 (IQR: 1.0-2.0), and 2019: 1.0 (IQR: 1.0-2.0). Similarly, the rate of outpatient procedures has been steadily increasing (2015: 8.3\%, 2016: 14.7\%, 2017: 15.8\%, 2018: 26.5\%, 2019: $34.6 \% ; \mathrm{P}<0.001)$.

Table 2

Unmatched comparison of outcomes between inpatient and outpatient shoulder arthroplasty $(\mathrm{N}=22,542)$.

\begin{tabular}{|c|c|c|c|c|c|}
\hline \multirow[t]{2}{*}{ Outcomes } & \multicolumn{2}{|c|}{$\begin{array}{l}\text { Inpatient } \\
\mathrm{N}=20,357\end{array}$} & \multicolumn{2}{|c|}{$\begin{array}{l}\text { Outpatient } \\
\mathrm{N}=2185\end{array}$} & \multirow[t]{2}{*}{$P$} \\
\hline & $\mathrm{N}$ & $\%$ & $\mathrm{~N}$ & $\%$ & \\
\hline Any adverse event & 1085 & $5.3 \%$ & 77 & $3.5 \%$ & $<0.001$ \\
\hline Minor adverse event & 613 & $3.0 \%$ & 33 & $1.5 \%$ & 0.001 \\
\hline Severe adverse event & 575 & $2.7 \%$ & 49 & $2.2 \%$ & 0.115 \\
\hline Readmission & 600 & $2.8 \%$ & 46 & $2.2 \%$ & 0.025 \\
\hline Reoperation & 293 & $1.4 \%$ & 22 & $1.0 \%$ & 0.102 \\
\hline
\end{tabular}

Bold indicates significance.
Table 3

Comparison of patient characteristics between inpatient and outpatient shoulder arthroplasty procedures following propensity score matched analysis $(\mathrm{N}=4344)$.

\begin{tabular}{|c|c|c|c|c|c|}
\hline \multirow[t]{2}{*}{ Variables } & \multicolumn{2}{|c|}{ Inpatient $N=2172$} & \multicolumn{2}{|c|}{ Outpatient $\mathrm{N}=2172$} & \multirow[t]{2}{*}{$\mathrm{P}$} \\
\hline & $\mathrm{N}$ & $\%$ & $\mathrm{~N}$ & $\%$ & \\
\hline Gender & & & & & 1.000 \\
\hline Female & 1069 & $49.2 \%$ & 1069 & $49.2 \%$ & \\
\hline Male & 1103 & $50.8 \%$ & 1103 & $50.8 \%$ & \\
\hline Race & & & & & 0.136 \\
\hline $\begin{array}{c}\text { Black/African } \\
\text { American }\end{array}$ & 89 & $4.1 \%$ & 110 & $5.1 \%$ & \\
\hline White & 1811 & $83.4 \%$ & 1820 & $83.8 \%$ & \\
\hline Other & 272 & $12.5 \%$ & 242 & $11.1 \%$ & \\
\hline ASA & & & & & 1.000 \\
\hline $1 \& 2$ & 1103 & $50.8 \%$ & 1103 & $50.8 \%$ & \\
\hline $3 \& 4$ & 1069 & $49.2 \%$ & 1069 & $49.2 \%$ & \\
\hline Age $\geq 65$ years & 1369 & $63.0 \%$ & 1369 & $63.0 \%$ & 1.000 \\
\hline Obesity & 1002 & $46.1 \%$ & 1002 & $46.1 \%$ & 1.000 \\
\hline Hypertension & 1312 & $60.4 \%$ & 1312 & $60.4 \%$ & 1.000 \\
\hline Diabetes mellitus & 323 & $14.9 \%$ & 323 & $14.9 \%$ & 1.000 \\
\hline COPD & 101 & $4.7 \%$ & 101 & $4.7 \%$ & 1.000 \\
\hline Congestive heart failure & 14 & $0.6 \%$ & 7 & $0.3 \%$ & 0.126 \\
\hline Weight loss $(>10 \%)$ & 0 & $0.0 \%$ & 5 & $0.2 \%$ & 0.025 \\
\hline Bleeding disorders & 26 & $1.2 \%$ & 26 & $1.2 \%$ & 1.000 \\
\hline Steroid use & 73 & $3.4 \%$ & 73 & $3.4 \%$ & 1.000 \\
\hline
\end{tabular}

ASA, American Society of Anesthesiologists.

Bold indicates significance.

\section{Discussion}

The most important finding of this study is that large, national multiyear data indicate that shoulder arthroplasty can be performed as safely in the outpatient setting as it can in the inpatient setting. When controlling for differences in patient comorbidities using propensity scorematched analysis, there were no significant differences in any-, minor-, or severe adverse events, readmission, and reoperation between inpatients and outpatients.

When comparing inpatient versus outpatient procedures, outpatient cases were more likely to be male and younger (age $<65$ years) and less likely to be ASA class 3 or 4, rather than 1 or 2 . Outpatients were also less likely to have various comorbidities including obesity, hypertension, diabetes mellitus, COPD, CHF, bleeding disorders, and chronic steroid use. These findings indicate that when insurance is not the driving factor dictating the decision to perform shoulder arthroplasty as outpatient or inpatient, shoulder surgeons are likely reserving outpatient procedures for more robust patients absent of significant comorbidities associated with increased morbidity and mortality [30-32]. These findings may mitigate concern that patient comorbidities drive procedure type. These findings are consistent with prior studies that advocate for the proper selection of patients for outpatient shoulder arthroplasty [26-28]. As advances in perioperative care continue to be established, the number of patients chosen for outpatient surgery will likely continue to rise and include patients of greater comorbidities. Further research is needed to identify what factors most greatly increase the risk of patient complications in the outpatient setting.

While outpatient procedures displayed decreased rates of adverse

Table 4

Risk-matched comparison of outcomes between inpatient and outpatient shoulder arthroplasty $(\mathrm{N}=4374)$.

\begin{tabular}{|c|c|c|c|c|c|}
\hline \multirow[t]{2}{*}{ Outcomes } & \multicolumn{2}{|c|}{$\begin{array}{l}\text { Inpatient } \\
\mathrm{N}=2172\end{array}$} & \multicolumn{2}{|c|}{$\begin{array}{l}\text { Outpatient } \\
\mathrm{N}=2172\end{array}$} & \multirow[t]{2}{*}{$\mathrm{P}$} \\
\hline & $\mathrm{N}$ & $\%$ & $\mathrm{~N}$ & $\%$ & \\
\hline Any adverse event & 88 & $4.1 \%$ & 76 & $3.5 \%$ & 0.339 \\
\hline Minor adverse Event & 43 & $2.0 \%$ & 32 & $1.5 \%$ & 0.200 \\
\hline Severe adverse event & 55 & $2.5 \%$ & 49 & $2.3 \%$ & 0.551 \\
\hline Readmission & 57 & $2.6 \%$ & 45 & $2.1 \%$ & 0.229 \\
\hline Reoperation & 37 & $1.7 \%$ & 22 & $1.0 \%$ & 0.066 \\
\hline
\end{tabular}


events in the non-risk-matched analysis, it was evident that this may be an artifact of patient selection. Following the propensity score matched analysis, there was no difference in the number of any-, minor-, or severe adverse events, as well as readmissions and reoperations between inpatient and outpatient procedures This analysis substantiates the equivalent outcomes previously displayed by single-institution analyses demonstrating the safety and efficacy of outpatient shoulder arthroplasty [5-14].

Despite demonstrating similar outcomes in movement and function, a recent study revealed that outpatient arthroplasty might be associated with an increased post-operative fall rate, as their single-institution experience demonstrated a significant difference (10.6\% vs. $1.0 \%)$. In addition to other adverse outcomes, patient fall rates may be diminished and eventually eliminated through careful monitoring and implementation of web-based home therapy programs. These programs have shown promise as supplements to the standard post-operative care seen with inpatient shoulder arthroplasty [33].

From 2015 to 2019, the cohort demonstrated a four-fold increase in outpatient shoulder arthroplasty. Outpatient procedures accounted for $34.6 \%$ of shoulder arthroplasties from the ACS-NSQIP database in 2019. This is a substantial increase since the only previous NSQIP study comparing outpatient and inpatient outcomes, which encompassed patients from 2005 to 2014, with only $2.4 \%$ of patients undergoing outpatient procedures [16]. Considering the equivalent outcomes of risk-match patients receiving inpatient and outpatient shoulder arthroplasty, it is expected the proportion of patients receiving outpatient procedures will continue to increase. Additionally, a recent survey study of outpatient shoulder arthroplasty patients revealed all patients were satisfied with their functional outcome and $84 \%$ were satisfied with their outpatient management specifically [34].

From a financial standpoint, these findings are important as health care policy makers have placed increased attention on the cost of health care, making outpatient arthroplasty a cost-effective alternative to routine hospital admission [4]. When compared to inpatient management for shoulder arthroplasty, performing the procedure in the ambulatory setting was associated with an average cost saving of $\$ 5,600$ (range: \$747-\$15,507) [23]. A recent study used this average cost difference and a predicted $36 \%$ outpatient procedure volume over the next ten years to calculate potential savings, which equated to $\$ 4.1$ million to $\$ 349$ million per year, and a ten year saving of $\$ 51$ million to $\$ 5.4$ billion [23]. It is likely that cost savings will continue to be a major driver in the continued increased proportion of shoulder arthroplasty cases performed as outpatient procedures.

There are several limitations to this study. First and foremost, the NSQIP database is not fully representative of the surgical environment of the United States, as the database is predominated by hospitals, with limited inclusion of outpatient surgery centers. Using these data may underestimate the occurrence of outpatient shoulder arthroplasty, across the United States. Moreover, NSQIP does not identify planned outpatient shoulder arthroplasty cases that ultimately were not discharged and required admission to the hospital. This study also lacks important range of motion and functional outcome scores (e.g. American Shoulder and Elbow Surgeons, single assessment numeric evaluation, and visual analog score) and any outcomes beyond 30-days.

\section{Conclusion}

From 2015 to 2019, there has been a four-fold increase in the proportion of outpatient shoulder arthroplasty cases in the ACS-NSQIP database. This study shows that outpatient shoulder arthroplasty may be safely performed in a select cohort of patients without increased risk of adverse events. After adjusting for comorbidities, there were no differences in clinical outcomes or rates of adverse outcomes between inpatient and outpatient shoulder arthroplasty.

\section{Author contributions}

All authors contributed to data analysis, drafting or revising the article, have agreed on the journal to which the article will be submitted, gave final approval of the version to be published and agree to be accountable for all aspects of the work.

\section{Declaration of competing interest}

The authors Trudeau MT, Peters JP, LeVasseur MR, Hawthorne BC, Dorsey CG, Wellington IJ, and Shea KP declare no conflicts of interest. Mazzocca $\mathrm{AD}$ reports research grants from Arthrex, Inc. and is a consultant for Arthrex, Inc.

\section{References}

[1] Ode GE, Odum S, Connor PM, Hamid N. Ambulatory versus inpatient shoulder arthroplasty: a population-based analysis of trends, outcomes, and charges. JSES Int 2020;4(1):127-32. https://doi.org/10.1016/j.jses.2019.10.001.

[2] Goltz DE, Burnett RA, Wickman JR, et al. Short stay after shoulder arthroplasty does not increase 90-day readmissions in Medicare patients compared to privatelyinsured patients. J Shoulder Elbow Surg 2022;31(1):35-42. https://doi.org/ 10.1016/j.jse.2021.05.013.

[3] Gallay SH, Lobo JJA, Baker J, Smith K, Patel K. Development of a regional model of care for ambulatory total shoulder arthroplasty: a pilot study. Clin Orthop Relat Res 2008;466(3):563-72. https://doi.org/10.1007/s11999-007-0083-5.

[4] Brolin TJ, Throckmorton TW. Outpatient shoulder arthroplasty. Orthop Clin North Am 2018;49(1):73-9. https://doi.org/10.1016/j.ocl.2017.08.011.

[5] Stone MA, Singh P, Rosario SL, Omid R. Outpatient total elbow arthroplasty: 90-day outcomes. J Shoulder Elbow Surg 2018;27(7):1311-6. https://doi.org/10.1016/ j.jse.2018.03.019.

[6] Charles MD, Cvetanovich G, Sumner-Parilla S, Nicholson GP, Verma N, Romeo AA. Outpatient shoulder arthroplasty: outcomes, complications, and readmissions in 2 outpatient settings. J Shoulder Elbow Surg 2019;286S):S118-23 https://doi.org/ 10.1016/j.jse.2019.04.006.

[7] Bean BA, Connor PM, Schiffern SC, Hamid N. Outpatient shoulder arthroplasty at an ambulatory surgery center using a multimodal pain management approach. J Am Acad Orthop Surg Glob Res Rev 2018;2(10):e064. https://doi.org/10.5435/ JAAOSGlobal-D-18-00064.

[8] Erickson BJ, Shishani Y, Jones S, et al. Outpatient vs. inpatient reverse total shoulder arthroplasty: outcomes and complications. J Shoulder Elbow Surg 2020; 29(6):1115-20. https://doi.org/10.1016/j.jse.2019.10.023.

[9] Leroux TS, Zuke WA, Saltzman BM, et al. Safety and patient satisfaction of outpatient shoulder arthroplasty. JSES Open Access 2018;2(1):13-7. https:// doi.org/10.1016/j.jses.2017.11.002.

[10] Arshi A, Leong NL, Wang C, et al. Relative complications and trends of outpatient total shoulder arthroplasty. Orthopedics 2018;41(3):e400-9. https://doi.org/ 10.3928/01477447-20180409-01.

[11] Harrison AK, Throckmorton TW, Shumate DH, Braman JP. Outpatient and semioutpatient total shoulder replacement: patient selection and program management. Instr Course Lect 2020;69:575-82. pmid: 32017752.

[12] Willenbring TJ, DeVos MJ, Kozemchak AM, Warth RJ, Gregory JM. Is outpatient shoulder arthroplasty safe in patients aged $\geq 65$ years? A comparison of readmissions and complications in inpatient and outpatient settings. J Shoulder Elbow Surg 2021;30(10):2306-11. https://doi.org/10.1016/j.jse.2021.02.022.

[13] Kramer JD, Chan PH, Prentice HA, Hatch J, Dillon MT, Navarro RA. Same-day discharge is not inferior to longer length of in-hospital stay for 90-day readmissions following shoulder arthroplasty. J Shoulder Elbow Surg 2020;29(5):898-905. https://doi.org/10.1016/j.jse.2019.09.037.

[14] Antonacci CL, Cu BJ, Erickson BJ, Vazquez O, Alberta FG. Complications and readmissions after reverse and anatomic total shoulder arthroplasty with same-day discharge. J Am Acad Orthop Surg 2021;29(3):116-22. https://doi.org/10.5435/ JAAOS-D-20-00245.

[15] Harris AB, Best MJ, Weiner S, Gupta HO, Jenkins SG, Srikumaran U. Hospital readmission rates following outpatient versus inpatient shoulder arthroplasty. Orthopedics 2021;44(2):e173-7. https://doi.org/10.3928/01477447-2020092503.

[16] Leroux TS, Basques BA, Frank RM, et al. Outpatient total shoulder arthroplasty: a population-based study comparing adverse event and readmission rates to inpatient total shoulder arthroplasty. J Shoulder Elbow Surg 2016;25(11):1780-6. https:// doi.org/10.1016/j.jse.2016.04.006.

[17] Steinhaus ME, Liu JN, Gowd AK, et al. The feasibility of outpatient shoulder arthroplasty: risk stratification and predictive probability modeling. Orthopedics 2021;44(2):e215-22. https://doi.org/10.3928/01477447-20201216-01.

[18] Basques BA, Erickson BJ, Leroux T, et al. Comparative outcomes of outpatient and inpatient total shoulder arthroplasty: an analysis of the Medicare dataset. Bone Joint J 2017;99-B(7):934-8. https://doi.org/10.1302/0301-620X.99B7.BJJ-20160976.R1. 
[19] Huddleston HP, Mehta N, Polce EM, et al. Complication rates and outcomes after outpatient shoulder arthroplasty: a systematic review. JSES Int 2021;5(3):413-23. https://doi.org/10.1016/j.jseint.2020.11.005.

[20] Ahmed AF, Hantouly A, Toubasi A, et al. The safety of outpatient total shoulder arthroplasty: a systematic review and meta-analysis. Int Orthop 2021;45(3): 697-710. https://doi.org/10.1007/s00264-021-04940-7.

[21] Cimino AM, Hawkins JK, McGwin G, Brabston EW, Ponce BA, Momaya AM. Is outpatient shoulder arthroplasty safe? A systematic review and meta-analysis. J Shoulder Elbow Surg 2021;30(8):1968-76. https://doi.org/10.1016/ j.jse.2021.02.007.

[22] Vajapey SP, Contreras ES, Neviaser AS, Bishop JY, Cvetanovich GL. Outpatient tota shoulder arthroplasty: a systematic review evaluating outcomes and costeffectiveness. JBJS Rev 2021;9(5). https://doi.org/10.2106/JBJS.RVW.20.00189.

[23] Steinhaus ME, Shim SS, Lamba N, Makhni EC, Kadiyala RK. Outpatient total shoulder arthroplasty: a cost-identification analysis. J Orthop 2018;15(2):581-5. https://doi.org/10.1016/j.jor.2018.05.038.

[24] Gregory JM, Wetzig AM, Wayne CD, Bailey L, Warth RJ. Quantification of patientlevel costs in outpatient total shoulder arthroplasty. J Shoulder Elbow Surg 2019; 28(6):1066-73. https://doi.org/10.1016/j.jse.2018.10.006.

[25] Walters JD, Walsh RN, Smith RA, Brolin TJ, Azar FM, Throckmorton TW. Bundled payment plans are associated with notable cost savings for ambulatory outpatient total shoulder arthroplasty. J Am Acad Orthop Surg 2020;28(19):795-801. https:// doi.org/10.5435/JAAOS-D-19-00441.

[26] Polisetty TS, Grewal G, Drawbert H, Ardeljan A, Colley R, Levy JC. Determining the validity of the Outpatient Arthroplasty Risk Assessment (OARA) tool for identifying patients for safe same-day discharge after primary shoulder arthroplasty. J Shoulder Elbow Surg 2021;30(8):1794-802. https://doi.org/10.1016/j.jse.2020.10.036.
[27] Fournier MN, Brolin TJ, Azar FM, Stephens R, Throckmorton TW. Identifying appropriate candidates for ambulatory outpatient shoulder arthroplasty: validation of a patient selection algorithm. J Shoulder Elbow Surg 2019;28(1): 65-70.

[28] Biron DR, Sinha I, Kleiner JE, et al. A novel machine learning model developed to assist in patient selection for outpatient total shoulder arthroplasty. J Am Acad Orthop Surg 2020;28(13):e580-5. https://doi.org/10.5435/JAAOS-D-19-00395.

[29] Austin PC. A critical appraisal of propensity-score matching in the medical literature between 1996 and 2003. Stat Med 2008;27(12):2037-49. https:// doi.org/10.1002/sim.3150.

[30] Belmont Jr PJ, Kusnezov NA, Dunn JC, Bader JO, Kilcoyne K, Waterman BR. Predictors of hospital readmission after total shoulder arthroplasty. Orthopedics 2017;40(1):e1-10. https://doi.org/10.3928/01477447-20160915-06.

[31] Menendez ME, Baker DK, Fryberger CT, Ponce BA. Predictors of extended length of stay after elective shoulder arthroplasty. J Shoulder Elbow Surg 2015;24(10):1527-33. https://doi.org/10.1016/j.jse.2015.02.014.

[32] Traven SA, McGurk KM, Reeves RA, Walton ZJ, Woolf SK, Slone HS. Modified frailty index predicts medical complications, length of stay, readmission, and mortality following total shoulder arthroplasty. J Shoulder Elbow Surg 2019; 28(10):1854-60. https://doi.org/10.1016/j.jse.2019.03.009.

[33] Davis DE, Cox R, Patel MS, Lazarus M, Ramsey M, Namdari S. Successful outcomes achieved via web-based, home program after total shoulder arthroplasty. Arch Bone Jt Surg 2020;8(6):661-7. https://doi.org/10.22038/abjs.2020.42832.2164.

[34] Cointat C, Gauci MO, Azar M, Tran L, Trojani C, Boileau P. Outpatient shoulder prostheses: feasibility, acceptance and safety. Orthop Traumatol Surg Res 2021; 107(4):102913. https://doi.org/10.1016/j.otsr.2021.102913. 